\title{
Assessment of Fasting Blood Plasma Glucose Level in Menopausal Women in Rural Area of Vom and Environs
}

\author{
Duru $\mathrm{BN}^{1^{*}}$., Omonayin $\mathrm{OJ}^{2}$., Gadzama $\mathrm{HM}^{2}$., Olawuyi $\mathrm{NO}^{2}$., Diddam $\mathrm{MA}^{2}$., \\ Nambol $\mathrm{DL}^{2}$., Ujah $\mathrm{AE}^{2}$., Ashi $\mathrm{RR}^{3}$., Okoye $\mathrm{NO}^{3}$., Tyem $\mathrm{DD}^{3}$., Udom $\mathrm{IE}^{3}$., \\ Salako $\mathrm{CY}^{3}$., Adeyanju $\mathrm{ON}^{3}$., Gushe $\mathrm{DD}^{4}$., Abilu $\mathrm{CJ}^{4}$., Danjuma $\mathrm{LA}^{4}$. \\ ${ }^{1}$ (Department of Chemical Pathology, F C V M L T, Vom) \\ 2 (Department of Chemical Pathology, F C V M L T, Vom) \\ ${ }^{2}$ (Department of Heamatology/BGS, F C V M L T, Vom) \\ 2 (Department of Chemical Pathology, F C V M L T, Vom) \\ ${ }^{2}$ (Viral Vaccine Production, $N$ V R I, Vom) \\ ${ }^{3}$ (Department of Histology, F C V M L T, Vom) \\ ${ }^{3}$ (Department of Livestock Investigations, NVRI, VOM) \\ ${ }^{3}$ (Department of Haematology/BGS, F C V M L T, Vom) \\ ${ }^{3}$ (Applied Biotechnology Division, NVRI, Vom) \\ ${ }^{3}$ (Department of Biochemistry F C A H \& P T, VOM) \\ ${ }^{3}$ (Department of Haematology/BGS, F C V M L T, Vom) \\ ${ }^{4}$ (Department of Biochemistry F C A H \& P T, VOM) \\ ${ }^{4}$ (Central Diagnostic Divition, N V R I, Vom) \\ ${ }^{4}$ (Department of Dermatophilosis, N V R I, Vom)
}

\begin{abstract}
Vom and its environs were investigated for their fasting blood plasma glucose levels. Blood glucose level was determined by glucose oxidase method (Randox Glucose Reagent Kits) to ascertain if menopause augments fasting blood glucose. The mean blood glucose level of menopausal women was found to be $4.43 \pm 0.15 \mathrm{mmol} / \mathrm{L}$ while that of the non-menopausal women (control) was found to be $4.09 \pm 0.10 \mathrm{mmol} / \mathrm{L}$ respectively. When comparism was made using one way ANOVA, the fastingblood plasma glucose levels of both menopausal women and non menopausal women were not significant $(P>0.05)$, even though mean Fasting blood plasma glucose level of the menopausal women was slightly higher. However Comparism by age group made using the Post Hoc test shows that age group of 60years and above, shows a clinical significant difference $(P<0.05)$. This led to the conclusion that blood glucose level certainly increases in women around and after age of 60 and fasting plasma glucose level predict future risk of diabetes and other associated diseases in menopausal women.
\end{abstract}

Keywords: Menopause, Fasting blood plasma glucose, Diabetes, Future risk,

\section{Introduction}

Menopause is the end of menstruation and fertility, which is a natural biological event, not a disease or illness. But however, for some women, physical and emotional symptoms can be difficult.[1 ].

Menopause involves hormonal changes in the body that may cause physical symptoms as the ovaries stop producing female sex hormone (oestrogen and progesterone), oestrogen levels declines over a period of years which causes symptoms such as hot flashes, mood changes, cold hands and feet. Technically, menopause is considered complete, when a woman has not had her period for an entire year .[2 ] [3].

Menopause may occur because of surgical removal of ovaries (Ovariectomy) or uterus (hysterectomy) as a part of treatment for abnormal menstruation after age of 35 years. Usually females with short menstrual cycle attains menopause earlier than the female with longer cycle.[4]. On average, menopause occurs at age 51, but it varies from person to person [2] , although the process can begin anytime from age 40 to the late 50's (fifties)[ 4].

During menopause, profound changes occurs which affects emotional and physical health. At that time, series of metabolic changes can lead to blood sugar imbalance, and other serious health associated problems. A study that tracked metabolic changes in women as they progressed through menopause found that one out of six (6) women developed blood sugar imbalance. Estrogen can create blood sugar imbalance and increase in body fat storage, where as progesterone normalize blood sugar levels and helps use fat for energy[ 5]

Blood sugar imbalance, if unchecked stimulates weight gain and metabolic dysfunction, increasing the risk of diabetes and cardiovascular disease. When blood sugar imbalance arises, increase in body fat occurs around the stomach, higher insulin levels and lipid imbalance and lower levels of the good cholesterol (HDL) 
and higher levels of triglycerides occurs. The way to this, is to ensure that there is no weight gain during menopause and unhealthy increase in glucose and insulin[5 ]. Ketoacidosis is a life-threatening condition which needs immediate treatment. Symptoms include: shortness of breath, breath that smells fruity (such as pear drops), nausea and vomiting, and very dry mouth. Chronic hyperglycemia (high blood sugar) injures the heart in patients without a history of heart disease or diabetes and is strongly associated with heart attacks and death in subjects with no coronary heart disease or history of heart failure [ 6]

Insulin, glucagon and epinephrine maintain the glucose concentration in the blood within a fairly narrow interval under diverse conditions (feeding, fasting, severe exercise)[ 5].

In non-diabetic women, menopause, but not age, is an independent risk factor for elevated fasting plasma glucose levels. However, glucose metabolism is influenced by various genetic and environmental factors [7].

\section{$1.1 \quad$ Justification}

In women, prevalence of abnormal glucose metabolism is known to increase at menopause and Chronic hyperglycemia (high blood sugar) injures the heart in patients without a history of heart disease or diabetes and is strongly associated with heart attacks and death in subjects with no coronary heart disease or history of heart failure, hence, fasting plasma glucose level is therefore important for the prevention and monitoring of diabetes and other associated diseases.

\subsection{Aims and Objectives}

1. To determine blood glucose level in menopausal women.

2. To establish the prevalence of hyperglycemia in menopausal women.

3. To determine the age distribution

\subsection{Subject Selection}

\section{Materials And Method}

Menopause women from ages of 40 and above were the subject used. The subjects were rural women living in Vom and its environs.

\subsection{Sample Collection}

151 samples were collected. The subjects were told to fast overnight and were given questionnaires each. Tourniquet was tied at the upper arm and with cotton wool soaked in methylated spirit, the anti-cubital foci vein of each patient was sterilized. $2 \mathrm{mls}$ of venous blood was withdrawn by vein puncture using a New disposable syringe and needle for each patient. Each sample was transferred into a labeled anticoagulated bottle containing fluoride oxalate and was transferred into a clean dry centrifuged tube and was centrifuged at 3000 revolution per minute (rpm) for 5 minutes. The plasma was obtained with the aid of Pasteur pipette into another clean dry labeled container and analyzed immediately.

\subsection{Inclusion Criteria}

All subjects were women who had seen their menopause for more than 1year and above.

All the subjects used had no known medical condition and in their families.

\subsection{Exclusion Criteria}

Any subject found to have any known medical condition and in the family.

Subjects who are alcoholics and smokers

Subjects who had undergone any form of surgery especially on reproductive organs, liver and pancreas.

Subjects on hormone replacement therapy (HRT).

All obese subjects were excluded.

2.5 Estimation of blood glucose by glucose oxidase colorimetric method[8].

\subsubsection{Procedure}

To $1000 \mu$ of reagents, was added $10 \mu \mathrm{l}$ of samples and standard, mixed thoroughly and incubated for 10 minutes at $37^{\circ} \mathrm{C}$ using the incubator, the absorbance of the samples and standards were read against the reagent blank within 30 minutes at $500 \mathrm{~nm}$. 


\section{Results}

Table 1 Show The Mean, Standard Deviation And standard Error of Mean of Glucose Level Between Menopausal and Non-menopausal Women

\begin{tabular}{ccccccc}
\hline Status & Total Number & Mean $(\mathbf{m m o l} / \mathbf{L})$ & Std. Deviation & Std. Error & F-value & Remark \\
\hline & & & & & & \\
Menopause & 100 & 4.433 & 1.546 & 0.1535 & \multirow{2}{*}{2.258} & Not Significant \\
Non menopause & 51 & 4.0922 & 0.7186 & 0.1006 & & \\
\hline Total & $\mathbf{1 5 1}$ & $\mathbf{4 . 3 1 7 9}$ & $\mathbf{1 . 3 2 3 8}$ & $\mathbf{0 . 1 0 7 7}$ & & \\
\hline
\end{tabular}

Table 2 Shows the Mean, Standard Deviation and Standard Error of Mean and Comparism Between The Age Group of Menopausal and Non menopausal Women (Control)

\begin{tabular}{ccccccc}
\hline AGE & Total Number & Mean $(\mathbf{m m o l} / \mathbf{L})$ & Std. Deviation & Std. Error & F-value & Remark \\
\hline $21-30$ & 33 & 3.8478 & 0.7579 & 0.1319 & & Not Significant \\
$31-40$ & 18 & 4.1848 & 0.6447 & 0.1407 & 1.338 \\
$41-50$ & 29 & 4.2731 & 1.0255 & 0.2011 & \\
$51-60$ & 40 & 4.4500 & 1.3303 & 0.2103 & \\
$>60$ & 31 & 4.6452 & 2.0943 & 0.3761 & \\
\hline Total & $\mathbf{1 5 1}$ & $\mathbf{4 . 3 1 7 9}$ & $\mathbf{1 . 3 2 3 8}$ & $\mathbf{0 . 1 0 7 7}$ & \\
\hline
\end{tabular}

Table 3 Shows the comparism between the age group of the control and the menopausal women.

\begin{tabular}{|c|c|c|c|c|c|}
\hline & Age Group1 & Age Group2 & $\mathbf{T}$ & Std. Error & P value \\
\hline \multirow{8}{*}{ Non Menopuase } & \multirow{4}{*}{ 21-30 } & $31-40$ & 0.33723 & 0.36789 & 0.361 \\
\hline & & $41-50$ & -0.08823 & 0.34559 & 0.799 \\
\hline & & $51-60$ & -0.26515 & 0.30993 & 0.394 \\
\hline & & $>60$ & -0.46031 & 0.32964 & 0.165 \\
\hline & \multirow{4}{*}{$31-40$} & 21-30 & -0.33723 & 0.36789 & 0.361 \\
\hline & & $41-50$ & -0.42546 & 0.38667 & 0.273 \\
\hline & & $51-60$ & -0.60238 & 0.35515 & 0.092 \\
\hline & & $>60$ & $-0.79754(*)$ & 0.37247 & 0.034 \\
\hline \multirow{12}{*}{ Menopause } & \multirow{4}{*}{$41-50$} & 21-30 & 0.08823 & 0.34559 & 0.799 \\
\hline & & $31-40$ & 0.42546 & 0.38667 & 0.273 \\
\hline & & $51-60$ & -0.17692 & 0.332 & 0.595 \\
\hline & & $>60$ & -0.37208 & 0.35047 & 0.29 \\
\hline & \multirow{4}{*}{$51-60$} & 21-30 & 0.26515 & 0.30993 & 0.394 \\
\hline & & $31-40$ & 0.60238 & 0.35515 & 0.092 \\
\hline & & $41-50$ & 0.17692 & 0.332 & 0.595 \\
\hline & & $>60$ & -0.19516 & 0.31536 & 0.537 \\
\hline & \multirow{4}{*}{$>60$} & 21-30 & 0.46031 & 0.32964 & 0.165 \\
\hline & & $31-40$ & $0.79754(*)$ & 0.37247 & 0.034 \\
\hline & & $41-50$ & 0.37208 & 0.35047 & 0.29 \\
\hline & & $51-60$ & 0.19516 & 0.31536 & 0.537 \\
\hline
\end{tabular}

Key. * = significant difference

\section{Discussion}

Glucose metabolism is influenced by various genetic and environmental factors [7]. Chronic hyperglycemia (high blood sugar) injures the heart in patients without a history of heart disease or diabetes and is strongly associated with heart attacks and death in subjects with no coronary heart disease or history of heart failure [ 6], a situation which is possible in menopausal women who do not check their blood glucose levels. It was observed from the questionnaire used in this study that most of the women lacked balanced diet in their regular meal intake and seldom exercises except for some who still goes to farm

The average mean value of blood glucose level in menopausal women was $4.433 \pm 1.5346$, while that of non-menopausal women (control) was $4.0922 \pm 0.7186$ (TABLE1). This result indicated that the mean blood glucose level in the menopausal women slightly increased than that of the non-menopausal women and is in agreement with study carried out by[7], which states that, the prevalence of abnormal glucose metabolism is known to increase around and after age 50 considered to be a menopausal age. For one thing, the hormonal fluctuations that are common to perimenopause can affect blood glucose levels. For another, some symptoms of menopause are the same as or easily confused with the symptoms of high or low blood glucose levels, so the cause must be determined before corrective action can be taken. In addition, both diabetes and menopause raise a woman's risk of osteoporosis, so women with diabetes must be proactive about taking steps to keep their bones strong. Lack of sleep, whether related to menopause, stress, or something else, can disrupt diabetes control and menopause is often associated with weight gain, which can make blood glucose control more difficult [9].

Statistically, the plasma glucose level of both menopausal and non-menopausal women shows no significant difference $(\mathrm{P}>0.05$ ) using one way ANOVA (TABLE 2), but at age 60years and above the mean 
value shows an increase in blood glucose level compared to other age groups, which are as follows; 21-30 years (3.8478), 31-40years (4.1848), 41-50years (4.2731), 51-60years (4.4500) and 60years and above (4.6452) and is in concordance with the work done by Laurie,[7].

When paired group evaluation was done to confirm the existence of different blood glucose levels amongst different age groups (TABLE 3). There was no statistical difference in all the age groups compared, except that of 60years and above which shows significant difference $(\mathrm{P}<0.05)$. It has been observed that for women in menopause, there may be an increase of syndrome $\mathrm{X}$ (insulin resistance) symptoms due to metabolic changes, including fluctuations in adrenal and thyroid secretions. And, since insulin is a predominant hormone, the body may have trouble balancing the subsidiary hormones like estrogen, progesterone, and testosterone. The decrease of other hormones like estradiol can even trigger insulin resistance in some women[10]. In addition in menopause, with much attention directed towards the potential role of estrogen replacement in the maintenance and regulation of metabolic function and body composition in women, interventions with various estrogen replacement regimens have not demonstrated reproducible attenuation or reversal of menopause-associated adverse anthropomorphic changes, insulin resistance, and declines in functional capacity [11][12].

Furthermore, in a work done by Otsuki et a 1[13], univariate regression analysis demonstrated that age, body mass index, triglycerides level, low-density lipoprotein cholesterol level, and menopause status were associated with fasting plasma glucose level, whereas high-density lipoprotein cholesterol level was not. However, stepwise multivariate regression analysis showed that the independent risk factors for elevated fasting plasma glucose levels were body mass index, menopause, and triglycerides level, whereas age and low-density lipoprotein cholesterol level did not contribute to fasting plasma glucose levels. Hence menopause was implicated in both analyses making it seem an important factor to be seriously considered among others.

\section{Conclusion}

In conclusion from the from the result and analysis this work shows that blood glucose level certainly increases in menopause with age and not menopause increasing blood glucose level, because age is a factor to be considered during menopause.

\section{Recommendation}

Menopausal women in general and particularly those approaching age 60 may need to check their blood glucose levesl regularly, in addition such women may also need to reduce sugar intake, while dwelling more on vegetables and fruits accompanied by little or moderate exercise.

\section{References}

[1]. Zaborowska, E., Bryhildsen J., Damberg S et al., Effects of acupuncture, appliedRelaxation, estrogens, and placebo on hot flashes in postmenopausal women: an Analysis of the propective, parallel, randomized studies. Climacteric, 10(), (2007), 38-45.

[2]. Uebelhack R, Blohmer JU, Graubaum HJ, et al., Black cohosh and St. John's wort for climacteric complaints: a randomized trial. Obstet Gynecol. 107, 2006, 247-255.

[3]. Robien K, Cutler G.J, Lazovich, Sept, vitamin D intake and breast cancer risk in Postmenopausal women. The Iowa women's health study. Cancer causes controls, 18 (7). 2007, 775-782.

[4]. Sembulingam, K., Sembulingam P, Essentials of medical physiology (Fourth Edition) (New Delhi ;Jaypee brothers' Medical publishers LTD, 2006), Pg. 260-261, 454-455.

[5]. Carl A Burtis, Edward R. Ashwood, David E. Bruns (Sixth Edtion) Tietz fundamental of clinical Chemistry. (published by Elsevier, 2008). Pg 373-377, 792.

[6]. "Chronic hyperglycemia may lead to cardiac damage". Journal of the American College of Cardiology. Retrieved 3 February 2012.

[7]. Laurie Barclay, Medscape Medical News: Menopause 14(), 2007, 404-407.

[8]. Trinder P. Determination of blood glucose using an oxidase-peroxidase system with a non-carcinogenic chromogen. J Clin Pathol. Mar;22(2), 1969, 158-161.

[9]. Pat Dougherty and Joyce Green Pastors , Menopause What to Expect, How to Cope. http://www.diabetesselfmanagement.com/articles/sexual-health/menopause/all/, 2008.

[10]. Marcelle Pick, Insulin Resistance? What Is It And How We Can Heal http://www.womentowomen.com/insulin-resistance/insulinresistance-what-is-it-and-how-we-can-heal/2013.

[11]. Sites CK, L'Hommedieu GD, Toth MJ, Brochu M, Cooper BC, Fairhurst PA, The effect of hormone replacement therapy on body composition, body fat distribution, and insulin sensitivity in menopausal women: a randomized, double-blind, placebo-controlled trial. J Clin Endocrinol Metab 90(),2005, 2701-2707.

[12]. Casson P, Toth M, Johnson J, Stanczyk F, Casey C, Dixon M. Correlation of serum androgens with anthropometric and metabolic indices in healthy, nonobese postmenopausal women. J Clin Endocrinol Metab. 95(9), 2010, 4276-4282.

[13]. Otsuki M, Kasayama S, Morita S, Asanuma N, Saito H, Mukai M, Koga M, Menopause, but not age, is an independent risk factor for fasting plasma glucose levels in non diabetic women. Menopause. 2007 May-Jun;14(3), 2007, 404-7. 